\title{
Análisis de la deforestación en La Macarena, antes y después de los acuerdos de paz
}

\section{Analysis of deforestation in La Macarena before and after the peace agreements}

\author{
Jully Andrea Forero Riaño* ${ }^{1 *}$ y Manuel Francisco Polanco Puerta
}

Forero Riaño, J. A. y Polanco Puerta, M. F. (2021). Análisis de la deforestación en La Macarena, antes y después de los acuerdos de paz. Colombia Forestal, 24(2), 9-23.

Recepción: 3 de junio 2020

\section{Resumen}

La presente investigación analizó la deforestación antes y después de los acuerdos de paz en los territorios del Área de Manejo Especial de La Macarena -AMEM-, pertenecientes al municipio de La Macarena, Meta. La metodología utilizada permitió comparar el cambio de área de bosque de 2015 a 2018 del AMEM, gracias a la aplicación de tres ecuaciones de tasa de deforestación, el cálculo del porcentaje de incremento en deforestación y el desarrollo de encuestas. Como resultado, este fenómeno aumentó en las cuatro áreas: dos Parques Nacionales Naturales -PNN- y dos Distritos de Manejo Integrado DMI-, siendo el área más afectada el PNN Tinigua, con un incremento de deforestación de 1176.1 \%, a consecuencia de diversos factores: el contexto histórico-social de necesidades insatisfechas y la violencia y ausencia de figuras del Estado, que se suman a motores de deforestación como el acaparamiento de tierras producto del incumplimiento de los acuerdos de paz.

Palabras clave: Área de Manejo Especial de La Macarena -AMEM-, distrito de manejo integrado, incremento de deforestación, tasa de deforestación.
Aprobación: 12 de abril 2021

\begin{abstract}
This research analysed deforestation before and after the peace agreements in territories of "La Macarena Special Management Area" -AMEM-, in the municipality of La Macarena, Meta, Colombia. The methodology used compared the change in forest area from 2015 to 2018 in the AMEM areas with the application of three deforestation rate equations and the calculation of the percentage of increase in deforestation, as well as the development of surveys. As a result, deforestation increased in the four areas: two National Natural Parks -PNN- and two Integrated Management Districts -DMI-, being the most affected area the PNN Tinigua, with an increase in deforestation of $1176.1 \%$ as a result of different reasons: the historical-social context of unsatisfied needs and the violence and absence of state figures, which add to drivers of deforestation such as land grabbing as a result of non-compliance with the peace agreements.
\end{abstract}

Keywords: La Macarena special management area -AMEM-, integrated management district, increased deforestation, deforestation rate.

1 Universidad de Manizales, Manizales, Colombia.

*aforero79310@umanizales.edu.co. Autora para correspondencia. 


\section{INTRODUCCIÓN}

El Acuerdo Final para la Terminación del Conflicto y la Construcción de una Paz Estable y Duradera, firmado el 24 de noviembre de 2016 entre el Gobierno de Colombia y las Fuerzas Armadas Revolucionarias de Colombia - Ejército del Pueblo -FARC-EP- contempla la formalización masiva de la pequeña y mediana propiedad rural como posible alternativa para las comunidades asentadas en el territorio; en lo relacionado con el componente ambiental este incluye el cierre de la frontera agrícola y la protección de zonas de reserva mediante la recuperación comunitaria de bosques (Gobierno Nacional y FARC-EP, 2016). El acuerdo marca el punto de partida para la modificación de algunas dinámicas en los bosques de Colombia, que se caracterizan por su alta biodiversidad.

Nuestro país, uno de los más biodiversos en el mundo por $\mathrm{km}^{2}$, es el segundo con mayor biodiversidad a nivel de plantas y el primero en anfibios y peces de agua dulce (Moreno et al., 2016); además, cuenta con un alto número de especies endémicas, 8803 aproximadamente (Moreno et al., 2019), que lo ubica como uno de los países con los mayores endemismos (Andrade Correa, 2011) producto de la heterogeneidad interna, dada la suma de sus componentes climáticos y geográficos.

Sin embargo, la biodiversidad de Colombia se encuentra en decremento a causa de la deforestación (Instituto de Investigación de Recursos Biológicos Alexander von Humboldt, 1998, citado por Andrade, 2011) y es considerada como la principal amenaza de pérdida de biodiversidad a nivel mundial (Etter et al., 2006).

En el caso de la Amazonía, por ejemplo, como resultado de la presión demográfica (Andrade y Castro, 2012), las actividades económicas de los procesos poblacionales han tenido impacto en la degradación de los ecosistemas naturales (Armenteras et al., 2006). Y pese a que el Estado aprobó, con el Documento CONPES 4021 de 2020, una Política Nacional para el Control de la Deforestación y la Gestión Sostenible de los Bosques, su diagnóstico indica que las figuras de conservación y protección que limitan el uso del suelo como los resguardos indígenas y los $\mathrm{PNN}$, aunque han incidido favorablemente en la conservación, también han sufrido procesos de pérdida de bosque que pone en riesgo los servicios ecosistémicos (CONPES 4007 de 2020).

La deforestación, además de ser una de las principales causas de la pérdida de biodiversidad en los bosques naturales, es una de las que más genera gases de efecto invernadero (Rodríguez, 2007). Aparte de sus implicaciones frente al cambio climático, también genera problemáticas puntuales en los ecosistemas circundantes afectando negativamente, por ejemplo, a gasterópodos del suelo, disminuyendo así el contenido de materia orgánica e incrementando el PH y la temperatura (Blanco-Libreros et al., 2013).

Como posibles factores que aceleran la deforestación en Colombia se encuentran el cambio de reglas locales de uso del suelo, dada la presencia o ausencia de actores armados, y los desafíos en la implementación de los acuerdos de paz (IDEAM y MADS, 2018). Adicionalmente, los cultivos ilícitos son otra causa de este problema (Cárdenas-Torres, 2006).

Con el fin de cuantificar la deforestación, el Instituto de Hidrología, Meteorología y Estudios Ambientales de Colombia -IDEAM- desarrolló un protocolo de procesamiento digital que brinda información sobre la superficie deforestada: una publicación oficial de periodicidad anual (Galindo et al., 2014). Si se toma esta cifra anual en hectáreas de bosque transformado y se aplica la tasa anual de deforestación, es posible identificar los cambios de cobertura forestal en dos momentos diferentes (Puyravaud, 2003).

Para el caso del municipio de La Macarena en el departamento del Meta, se observa que confluyen una alta biodiversidad y una alta deforestación. Este municipio, además de hacer parte del AMEM, se encuentra en la zona oriental de la cordillera de los Andes, en la Orinoquía y la Amazonía, convergiendo en la Sierra de La Macarena, que se presume fue un centro de dispersión de flora y fauna para el continente (Idrobo, 1984). A lo largo de su historia, La Macarena ha sufrido del ciclo Ilamado colonización armada (Espinosa, 2006), aunque también se destaca por la actividad ganadera, causante de 


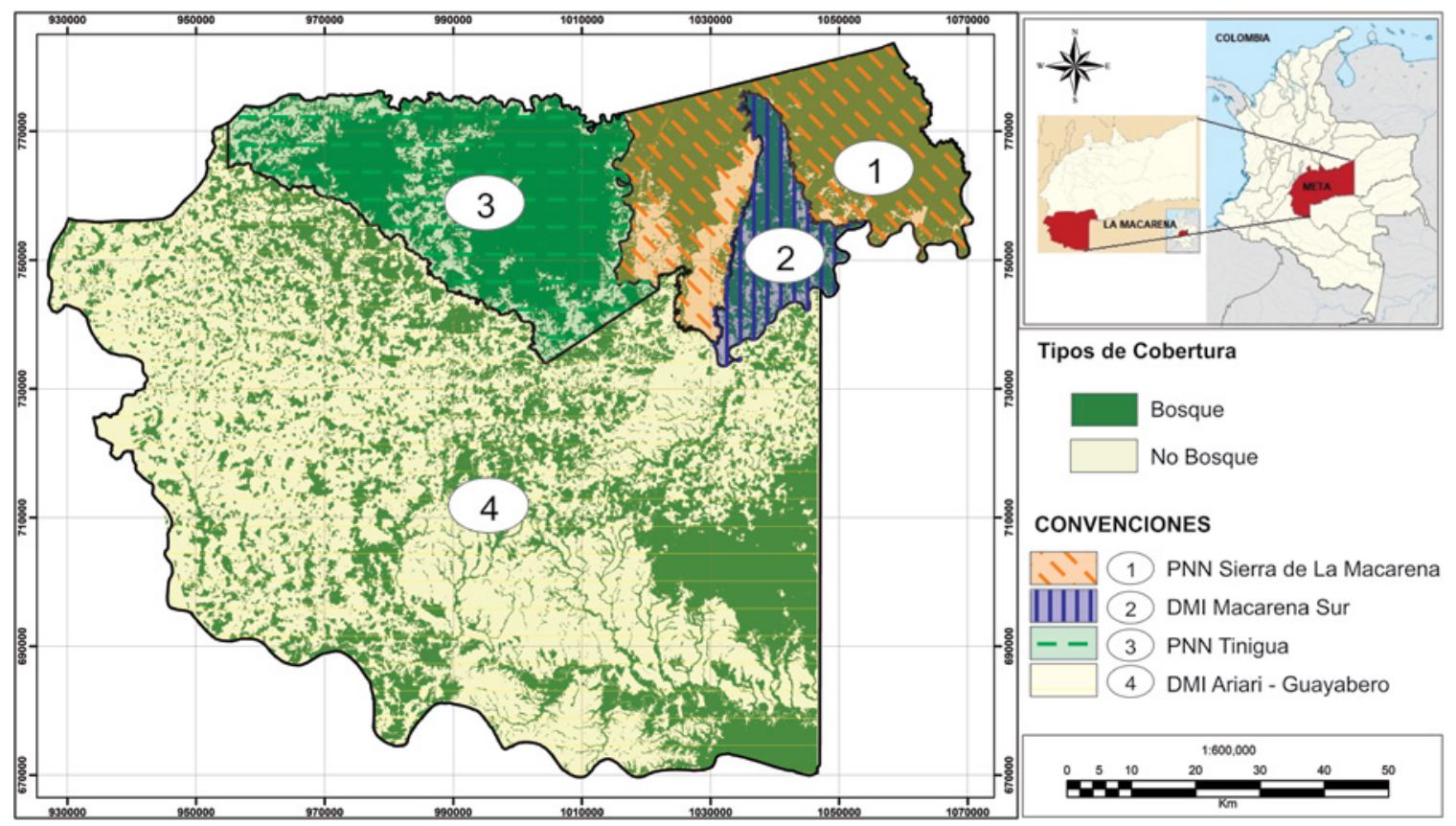

Figura 1. Área de estudio: Municipio de La Macarena, ubicado en el departamento del Meta.

Nota: hacen parte de este municipio el PNN Sierra de La Macarena, el PNN Tinigua, el DMI Ariari Guayabero y el DMI Macarena Sur. También se evidencia un área de bosque continuo en el PNN Tinigua. El DMI Ariari Guayabero es el área con mayor representatividad, contando con un porcentaje de ocupación del 70.09 \% como se observa en la Tabla 1.

la praderización y que afecta zonas de bosque muy importantes (CORMACARENA, 2016).

En las áreas boscosas de este municipio la colonización y la deforestación están ligadas. Conforme a lo indicado por Botero-García et al. (2019), para el AMEM la colonización se inició hacia la década de los años 30 del siglo pasado; luego se produjo una segunda etapa en la década de los 50, resultado de la violencia entre liberales y conservadores; posteriormente, se desarrolló un tercer proceso de colonización en la década de los 80. Cabe resaltar que, sumado a estos procesos, en el territorio hay presencia de grupos armados al margen de la ley, como el Bloque oriental de las FARC-EP con los frentes 40 y 7, y la columna móvil Luis Pardo (UNODC y MinJusticia, 2015).

Por lo anterior, en este trabajo se definió, como objetivo principal, analizar la deforestación en las áreas del AMEM del municipio de La Macarena entre 2015 y 2018, dos años antes y dos años después de los acuerdos de paz, y como objetivos específicos: i) determinar si hubo cambio en la tendencia de la deforestación en los periodos comprendidos entre
2015-2016 y 2017-2018 en las áreas del AMEM presentes en La Macarena, al aplicar las tasas de deforestación, y ii) examinar la relación de los acuerdos de paz con los bosques en La Macarena.

\section{MATERIALES Y MÉTODOS}

\section{Unidad de análisis y ubicación}

El estudio se desarrolló en La Macarena, municipio que limita al sur y al occidente con el departamento de Caquetá; al oriente, con el departamento de Guaviare; y al norte, con los municipios de Vistahermosa, Mesetas y Uribe en el departamento del Meta (Figura 1). En su totalidad, este municipio hace parte del Área de Manejo Especial de La Macarena -AMEM-, de gran importancia biológica, ecológica y biogeográfica (Granados-Martínez et al., 2018), reconocida como reserva biológica de la humanidad por la Ley 52 de 1948. A su vez, el AMEM está conformado por cuatro Parques Nacionales Naturales [PNN] y tres Distritos de Manejo Integrado [DMI]. 
Tabla 1. Hectáreas y porcentaje de ocupación de los DMI y PNN en el municipio de La Macarena.

\begin{tabular}{lcc}
\hline \multicolumn{1}{c}{ Área del municipio de La Macarena } & Hectáreas & $\begin{array}{c}\text { Porcentaje (\%) de ocupación } \\
\text { en el municipio }\end{array}$ \\
\hline DMI Ariari Guayabero & 743388.18 & 70.09 \\
DMI Macarena Sur & 38399.47 & 3.62 \\
PNN Sierra de la Macarena & 125425.74 & 11.83 \\
PNN Tinigua & 153368.31 & 14.46 \\
\hline
\end{tabular}

\section{Fuentes de información}

Para aplicar el cálculo de las tasas de deforestación se emplearon los resultados de cambio de cobertura obtenidos de la información oficial para Colombia, la cual corresponde a la cuantificación brindada por el IDEAM a escala 1:100 000. Los productos en raster de la implementación del protocolo de procesamiento digital de imágenes para la cuantificación de la deforestación en Colombia, nivel nacional escala gruesa y fina (Cabrera et al., 2011), y del protocolo de procesamiento digital de imágenes para la cuantificación de la deforestación en Colombia (Galindo et al., 2014), se obtuvieron utilizando imágenes satelitales Landsat 7, Landsat 8 y Sentinel 2 en el software QGIS, que permitieron cuantificar las áreas afectadas por este fenómeno e identificar los sitios críticos de ocurrencia a nivel espacial durante un periodo determinado.

Se realizaron 23 encuestas semiestructuradas siguiendo la metodología de encuestas empleada por Carr (2003) en su estudio sobre colonización y deforestación en la Reserva de la Biosfera Maya. Para el caso del presente estudio el primer segmento de estas encuestas fue llevado a cabo de manera presencial el 6 de noviembre de 2018 en la escuela veredal de Getsemaní 1, en el marco de una reunión comunitaria a la cual asistieron representantes de las veredas Getsemaní 1, Getsemaní 2, La Batalla 2, El Verdugo, El Oasis, El Edén del Tigre y El Triunfo, y el segundo segmento, a consecuencia de dificultades de orden público y del estado de emergencia declarado a causa del COVID-19, se desarrolló a través de encuestas telefónicas a los presidentes de Juntas de Acción Comunal -JAC- y a líderes del listado otorgado por la Alcaldía municipal. Las encuestas presentaron tres componentes: 1 ) actividades económicas, 2) uso del bosque natural y 3) alternativas para la protección de los recursos naturales.

\section{Técnicas de análisis}

La investigación desarrollada tuvo un enfoque mixto, ya que se realizó un análisis de la pérdida de bosques en cada una de las figuras ambientales que componen el municipio (PNN Tinigua, PNN Sierra de La Macarena, DMI Ariari Guayabero y DMI Macarena Sur) en los dos años previos y los dos años posteriores a la firma del acuerdo de paz; estos resultados se complementan con el empleo de técnicas de recolección de información por medio de 23 encuestas realizadas a presidentes de las JAC y a líderes del municipio, y la revisión del contexto social y de los acuerdos de paz firmados en Colombia en noviembre de 2016.

Respecto al análisis e identificación de los cambios de cobertura por pérdida de bosque en el municipio de La Macarena, se procedió a calcular las tasas de deforestación y su porcentaje de incremento con base en la información suministrada por el IDEAM, que corresponde a 9024 polígonos de deforestación en todo el municipio, equivalentes a 43 859.68 ha de bosque intervenido y transformado. Se emplearon como categorías las cuatro áreas del AMEM presentes en el municipio (dos PNN y dos DMI) y su relación en los dos años previos y los dos posteriores a la firma de los acuerdos de paz, utilizando el test no paramétrico de Mann-Whitney para evaluar la relación del tamaño de los polígonos, 
antes y después de la firma de los acuerdos. Esta información se contrastó con la observación directa y con los resultados del procesamiento de las encuestas, que contaron con tres secciones: la primera correspondió a las actividades económicas, tipo de ganadería y productos agrícolas; la segunda a la relación con el bosque natural; y la tercera a las alternativas de mitigación frente al daño ambiental, la cual, aunque proponía preguntas abiertas, presentó respuestas reiterativas por parte de los encuestados. Esta metodología es una variación de la empleada por Monjardín-Armenta et al. (2017).

Con el fin de evaluar los cambios en deforestación antes y después de la firma de los acuerdos de paz en las cuatro categorías ambientales, se calculó la tasa promedio anual de deforestación mediante la Ecuación 1 (IDEAM, 2002; Ruiz et al., 2011):

$$
T M A D=\frac{A B_{t 1}-A B_{t 2}}{n}
$$

Donde:

TMAD = tasa promedio anual de deforestación

$\mathrm{AB}_{\mathrm{t} 1}=$ área bosque momento 1

$\mathrm{AB}_{\mathrm{t} 2}=$ área bosque momento 2

$\mathrm{n}=$ diferencia de los años entre el momento $\mathrm{t} 1$ $y$ el momento $t 2$

Para determinar la tasa de deforestación en \%/ año se usó la Ecuación 2 empleada por Pozzobon y Osorio (2002), la cual expresa el porcentaje de área disminuida por año:

$$
T d=\left(\frac{\left(A B_{t 1}-A B_{t 2}\right)}{\left(A B_{t 1} * n\right)}\right) * 100
$$

Donde:

$\mathrm{Td}=$ tasa de deforestación por año (\%/año)

$\mathrm{AB}_{\mathrm{t} 1}=$ área bosque momento 1

$\mathrm{AB}_{\mathrm{t} 2}=$ área bosque momento 2

$\mathrm{n}=$ diferencia de los años entre el momento $\mathrm{t} 1$ y el momento $t 2$

Para el cálculo de la tasa de deforestación se utilizó la fórmula de tasa de deforestación empleada por la FAO [Organización de las Naciones Unidas para la Agricultura y la Alimentación] (1996)
(Ecuación 3) y la fórmula de tasa de deforestación propuesta por Puyravaud (2003) (Fórmula 4):

$$
\begin{gathered}
\operatorname{Td} F A O(r)=\left(\frac{A B_{t 2}}{A B_{t 1}}\right)^{\frac{1}{(t 2-t 1)}}-1 \\
\operatorname{Td} P(q) .=\frac{1}{\left(t_{2}-t_{1}\right)} * \ln \frac{A B_{t 2}}{A B_{t 1}}
\end{gathered}
$$

Donde:

Td FAO $(r)$ = tasa de deforestación empleada por la FAO (1996)

Td P. $(q)$ = tasa de deforestación empleada por Puyravaud (2003)

$\mathrm{AB}_{\mathrm{t} 1}=$ área bosque momento 1

$\mathrm{AB}_{\mathrm{t} 2}=$ área bosque momento 2

$\mathrm{n}=$ diferencia de los años entre el momento $\mathrm{t} 1$ y el momento $t 2$

$\mathrm{t}_{1}=$ año de inicio del momento 1

$\mathrm{t}_{2}=$ año de inicio del momento 2

Adicional a lo anterior, se propuso emplear la fórmula de incremento porcentual para obtener el resultado del porcentaje de aumento en deforestación, realizando la comparación de los años previos y posteriores a los acuerdos de paz. Para ello se utilizó la siguiente fórmula:

$$
\% I D=\left(\frac{\left(T M A D_{t 2}-T M A D_{t 1}\right)}{T M A D_{t 1}}\right) * 100
$$

$\%$ I.D. $=$ porcentaje de incremento de deforestación

$\mathrm{TMAD}_{\mathrm{t} 1}=$ tasa promedio anual de deforestación en el momento 1

$\mathrm{TMAD}_{\mathrm{t} 2}=$ tasa promedio anual de deforestación en el momento 2

\section{RESULTADOS}

\section{Análisis de deforestación antes y después de los acuerdos de paz}

Con las hectáreas y el número de polígonos de bosque natural perdidos de 2015 a 2018, obtenidos de la información oficial generada por el IDEAM para la cuantificación de la deforestación, se 
discriminaron las cuatro áreas del AMEM presentes en el municipio de La Macarena y se comparó el cambio en relación a dos periodos: dos años anteriores a la firma de los acuerdos de paz y dos años después de la firma, observando lo siguiente:

En la Figura 2 se presenta la cartografía con polígonos de deforestación antes y después de los acuerdos de paz en las cuatro categorías de manejo, evidenciando que aumentó la deforestación en este municipio después de los acuerdos de paz.
Con el fin de identificar el área que sufrió más pérdida de bosque natural, en la Tabla 2 se detallan las hectáreas pérdidas, así como la cantidad de polígonos en las áreas del AMEM presentes en el municipio antes y después de los acuerdos.

En la Tabla 2 se observa que aumentaron tanto las hectáreas como el número de polígonos después de los acuerdos de paz; sin embargo, no se evidencia que dicha relación sea directamente proporcional. Por tanto, se procedió a realizar la prueba del test

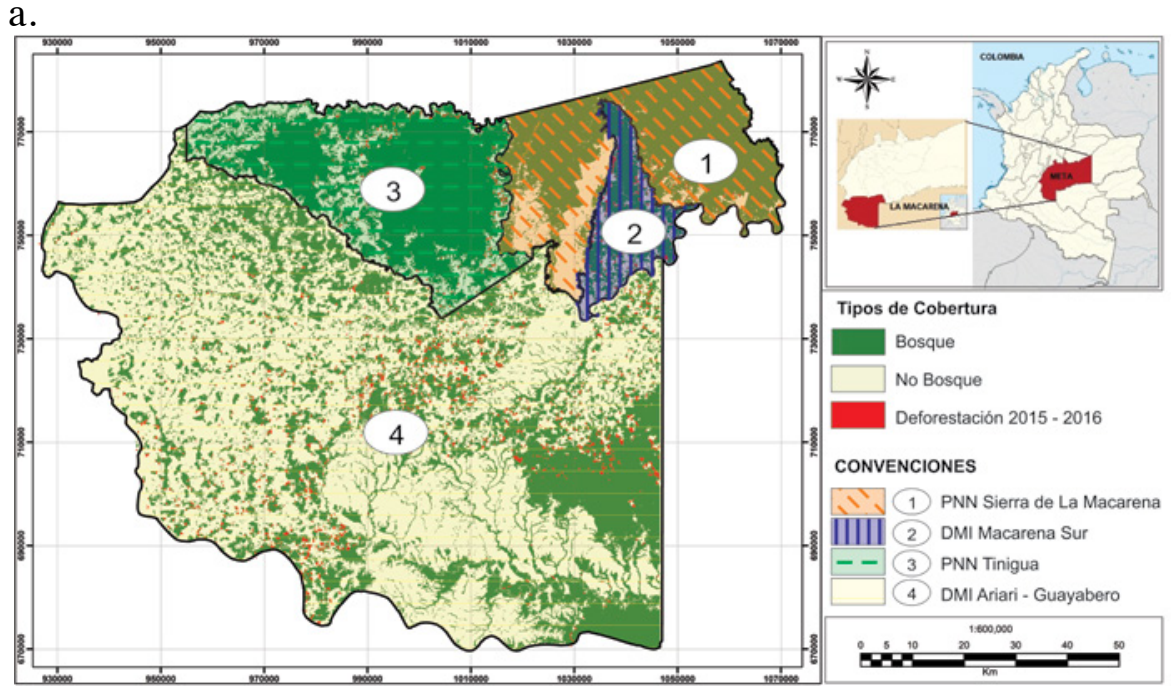

b.

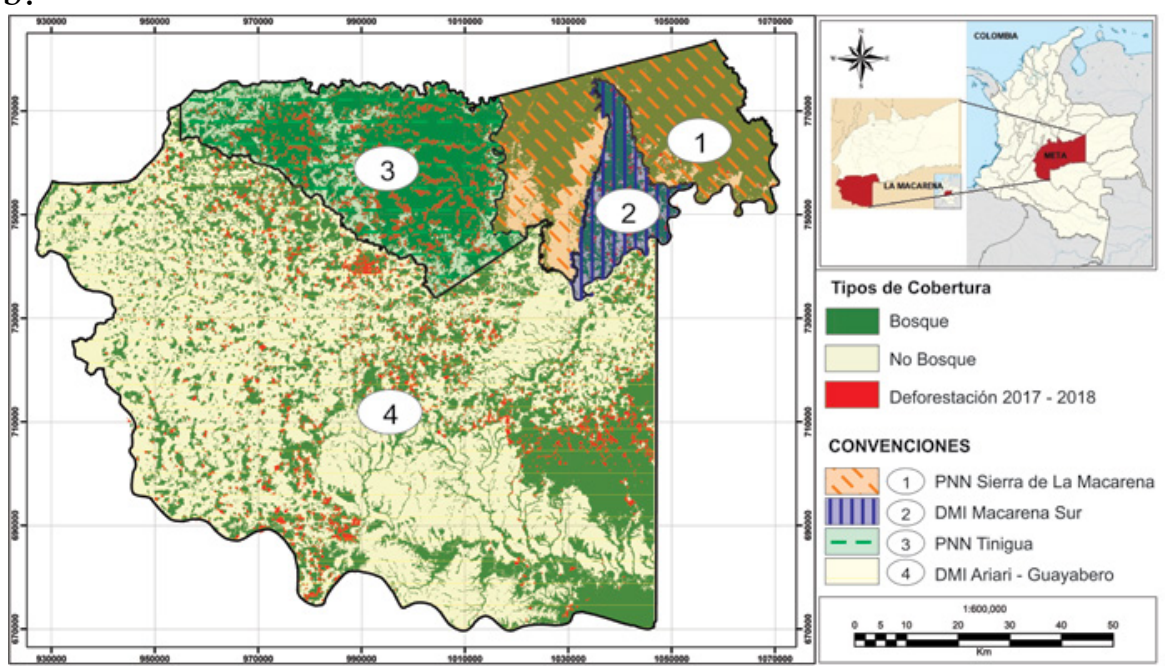

Figura 2. a. Deforestación antes de la firma de los acuerdos de paz; b. Deforestación después de la firma de los acuerdos de paz.

Nota: en las figuras están señaladas las cuatro áreas del AMEM presentes en el municipio: PNN Sierra de La Macarena (1), DMI Macarena Sur (2), PNN Tinigua (3) y DMI Ariari Guayabero (4). 
Tabla 2. Deforestación de las áreas del AMEM presentes en el municipio en los años previos y posteriores a los acuerdos de paz.

\begin{tabular}{lcccccc}
\hline \multicolumn{1}{c}{ Áreas de AMEM } & \multicolumn{2}{c}{ Antes de los Acuerdos } & Después de los Acuerdos & & \\
\hline & Def. (Ha) & \# Polígonos & Def. (Ha) & \# Polígonos & $\begin{array}{c}\text { Def. Total } \\
\text { (Ha) }\end{array}$ & $\begin{array}{c}\text { \# Polígonos } \\
\text { Totales }\end{array}$ \\
DMI Ariari Guayabero & 7565.56 & 2363 & 19555.1 & 4049 & 27121 & 6412 \\
DMI Macarena Sur & 701.39 & 307 & 1139.5 & 250 & 1841 & 557 \\
PNN Sierra de la Macarena & 249.34 & 111 & 478.1 & 112 & 727 & 223 \\
PNN Tinigua & 1029.76 & 302 & 13140.9 & 1530 & 14171 & 1832 \\
\hline
\end{tabular}

de Mann-Whitney-Wilcoxon, en la que se compararon las medianas de las áreas deforestadas con un resultado de $p=2.2 \mathrm{e}-16$, lo que permite confirmar que los polígonos deforestados son de mayor área después de la firma de los acuerdos de paz. El incremento de perdida de bosque natural en cada categoría de manejo del AMEM se observa en la Figura 3.

En la Figura 3 se observa el aumento de deforestación después de la firma de los acuerdos de paz, más visible en el PNN Tinigua y en el DMI Ariari Guayabero.

Al aplicar las fórmulas de tasa de deforestación y porcentaje de incremento para las cuatro áreas del AMEM presentes en La Macarena en los periodos previo y posterior a la firma de los acuerdos de paz, como se observa en la Tabla 3, se identificó un aumento drástico de pérdida de bosque natural en los años posteriores a los acuerdos.

Con base en la Tabla 3, se identificó un aumento considerable en la pérdida de bosque natural después de la firma de los acuerdos de paz. Los registros oficiales en los informes anuales de monitoreo a la deforestación exponen un incremento considerable de deforestación en los años posteriores a la firma de los acuerdos de paz con 5238 hectáreas de bosque perdido en 2016 (IDEAM y MADS, 2017), 11461 hectáreas perdidas en 2017 (IDEAM y MADS, 2018)

Deforestación antes y después de la firma de los acuerdos de paz

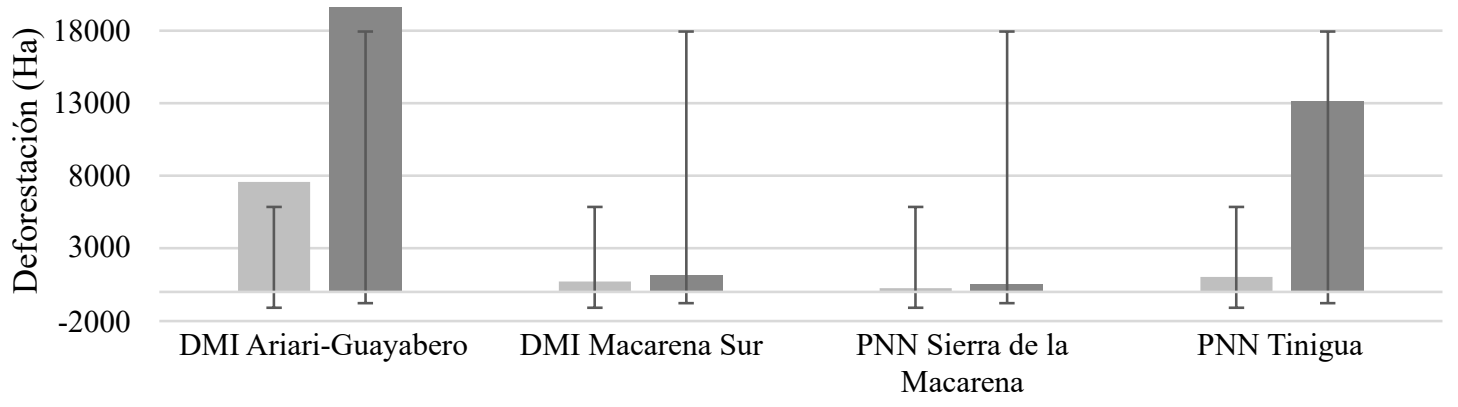

Categorias de manejo del Área de Manejo Especial la Macarena -AMEM-

$\square$ Antes de los acuerdos de paz $\quad$ Después de los acuerdos de paz

Figura 3. Relación de áreas deforestadas por categorías de manejo en el municipio de La Macarena, Meta, antes y después de la firma de los acuerdos de paz. 
Tabla 3. Tasa promedio anual de deforestación -TMAD- y Tasas de deforestación -Td- en las cuatro áreas del AMEM presentes en el municipio de La Macarena, antes y después de la firma de los acuerdos de paz.

\begin{tabular}{|c|c|c|c|c|c|c|c|c|c|}
\hline & $\begin{array}{c}\text { TMAD } \\
\text { A* }^{*}\end{array}$ & $\begin{array}{c}\text { TMAD } \\
\text { D }^{*}\end{array}$ & $\begin{array}{l}\text { Td \%/ } \\
\text { Año A* }\end{array}$ & $\begin{array}{l}\text { Td \%/ } \\
\text { Año D* }\end{array}$ & $\begin{array}{c}\text { Td FAO } \\
\text { A* }^{*}\end{array}$ & $\begin{array}{c}\text { Td Fao } \\
D^{*}\end{array}$ & Td P. A* & Td P. D* & $\%$ ID \\
\hline DMI Ariari Guayabero & 3782.8 & 9777.5 & 1.348 & 3.581 & -0.014 & -0.036 & -0.014 & -0.037 & 158.5 \\
\hline DMI Macarena Sur & 350.7 & 569.8 & 1.386 & 2.315 & -0.014 & -0.023 & -0.014 & -0.024 & 62.5 \\
\hline $\begin{array}{l}\text { PNN Sierra de la Ma- } \\
\text { carena }\end{array}$ & 124.7 & 239.1 & 0.127 & 0.244 & -0.001 & -0.002 & 0.001 & -0.002 & 91.8 \\
\hline PNN Tinigua & 514.9 & 6570.5 & 0.454 & 5.843 & -0.005 & -0.060 & -0.005 & -0.062 & 1176.1 \\
\hline
\end{tabular}

Nota: $\mathrm{A}^{*}$ : antes de los acuerdos de paz; $\mathrm{D}^{*}$ : después de los acuerdos de paz.

y 18680 hectáreas deforestadas en 2018 (IDEAM y MADS, 2019). El análisis a nivel de categorías de manejo del AMEM del presente estudio permitió identificar que las áreas más afectadas corresponden a: el PNN Tinigua, que pasó de una tasa media anual de 514.8 ha.año ${ }^{-1}$ a 6570.4 ha.año-1 ${ }^{-1}$ lo que indicó un crecimiento de $1176.1 \%$ en deforestación, y el DMI Ariari Guayabero, que pasó de 3782.7 ha.año ${ }^{-1}$ a una tasa de 9777.5 ha.año-1 ${ }^{-1}$ lo que indicó un crecimiento de $159 \%$ en deforestación con respecto a la tasa de deforestación FAO (r) y la tasa de deforestación empleada por Puyra$\operatorname{vaud}(q)$. Se evidencia la misma tendencia de incremento de la deforestación para todas las áreas después de los acuerdos.

\section{Análisis de las encuestas}

La deforestación está asociada, más que a la concentración poblacional, a las actividades económicas emanadas de la misma. Debido a esto, en la primera sección de las encuestas se buscó establecer qué relación existe entre la población y las actividades económicas, el tipo de ganadería y los productos agrícolas (Tabla 4); la segunda sección correspondió a la relación con el bosque natural (Tabla 5); y la tercera, a las alternativas de mitigación al daño ambiental, sección que, aunque proponía preguntas abiertas, evidenció respuestas reiterativas por parte de los encuestados.

Como se observa en la Tabla 4, la actividad que más se desarrolla en el municipio de La Macarena es la ganadería con un $46 \%$, seguida de la agricultura con $34.9 \%$. El tipo de ganadería que más se presenta en la zona es de mediana escala con 46 $\%$, y el producto agrícola que se siembra con mayor frecuencia es la yuca, que representa un $24 \%$.

Según la Tabla 5, se identifica que la madera para leña es el producto del bosque más usado con un $48 \%$, seguido de madera en bloque con

Tabla 4. Actividades económicas, tipo de ganadería y productos agrícolas en el municipio de La Macarena.

\begin{tabular}{ccccccc}
\hline & Actividad & Respuestas & Tipo de ganadería & Respuestas & Producto agrícola & Respuestas \\
\cline { 2 - 7 } & Ganadería & $46 \%$ & \multirow{2}{*}{ Mediana escala } & $46 \%$ & Yuca & $24 \%$ \\
& Agricultura & $34.90 \%$ & & & Plátano & $22 \%$ \\
& & & & Arroz & $16 \%$ \\
\hline
\end{tabular}


Tabla 5. Relación con el bosque natural en el municipio de La Macarena.

\begin{tabular}{|c|c|c|c|c|}
\hline \multirow{6}{*}{ 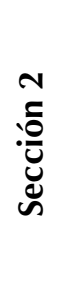 } & Empleo de productos del bosque & Respuestas & Recurso natural afectado & Respuestas \\
\hline & Madera para leña & $48.35 \%$ & Bosque & $40.48 \%$ \\
\hline & Madera en bloque & $27.55 \%$ & Agua & $28.57 \%$ \\
\hline & Carne de monte & $10.43 \%$ & Aire & $14.29 \%$ \\
\hline & Hojas para techo & $6.80 \%$ & Fauna & $9.52 \%$ \\
\hline & Frutos alimenticios & $6.80 \%$ & Suelo & $7.14 \%$ \\
\hline
\end{tabular}

un $27.55 \%$. Frente al recurso natural afectado por las actividades actuales, el $40.48 \%$ de los encuestados indicó que el bosque es el recurso más afectado, seguido del agua con $28.57 \%$.

Respecto a la última sección (las alternativas de mitigación al daño ambiental), en las propuestas para la conservación de los recursos naturales se formuló el siguiente interrogante: ¿qué alternativas considera que se pueden emplear para la protección de los recursos naturales? Aunque la pregunta fue abierta, la mayoría de la comunidad encuestada coincidió en que es necesario implementar proyectos productivos con maquinaria para los suelos; también en la necesidad de aumentar la cobertura mediante la reforestación y la creación de incentivos para la conservación; en menor medida indicaron que se requiere una política pública de ganadería intensiva y una adecuada titulación de predios.

\section{DISCUSIÓN}

Al calcular la tasa de deforestación se identificó que fue superior en todas las categorías de manejo del municipio de La Macarena en los años posteriores a los acuerdos; resultados que ratifican la tendencia de incremento de afectaciones ambientales en coberturas naturales presentada por con Armenteras et al. (2019), quienes informan sobre el aumento de incendios tras la desmovilización de la guerrilla en Colombia en las áreas protegidas evaluadas. Esta tendencia persiste en los resultados obtenidos por Murillo-Sandoval et al. (2020) acerca del aumento acelerado de la perturbación del área boscosa en toda la zona del cinturón de transición entre los Andes y la Amazonía. Frente a los resultados de la tasa de deforestación FAO (r) y la empleada por Puyra$\operatorname{vaud}(q)$, se evidencia la misma tendencia de incremento después de los acuerdos. En el PNN Tinigua y los DMI Ariari Guayabero y Macarena Sur se observó que la tasa $r$ es ligeramente superior a la tasa q, lo que coincide con los resultados obtenidos por Puyravaud (2003); sin embargo, las tasas de deforestación obtenidas en el presente estudio son superiores para el PNN Tinigua.

Al calcular el porcentaje de incremento en deforestación, se identificó que el área más afectada corresponde al PNN Tinigua donde la deforestación aumentó $1176.1 \%$, seguida del DMI Ariari Guayabero con $159 \%$, el PNN Sierra de La Macarena con $91.8 \%$ y el DMI Macarena Sur con $62.5 \%$. Estos resultados coinciden con los obtenidos por Murillo-Sandoval et al. (2020), quienes indicaron que el incremento en deforestación después de los acuerdos de paz es más evidente en las áreas protegidas, en especial en el PNN Tinigua. La información oficial brindada en los resultados de monitoreo a la deforestación para Colombia también reporta que en 6 áreas protegidas se concentra más del $80 \%$ de la deforestación del sistema de parques, ubicándose el PNN Tinigua como uno de los tres parques con mayor deforestación en La Macarena, siendo el tercero más deforestado en 2016 (IDEAM y MADS, 2017), el segundo más deforestado en 2017 (IDEAM y MADS, 2018) y el primero más deforestado en Colombia en 2018 (IDEAM y MADS, 2019).

En otros países, a consecuencia de la firma de acuerdos de paz entre diferentes actores armados, 
también se presentó un incremento en la tasa de deforestación -al igual que en Colombia-, como es el caso de Ruanda, República Democrática del Congo y Sierra Leona, lugares donde se generaron procesos de reasentamiento que terminaron acabando con miles de hectáreas de bosque, pues dichos acuerdos y su puesta en marcha no contemplaron el medioambiente, provocando así una crisis por los recursos y propiciando el surgimiento de nuevos grupos armados causantes de más inseguridad (Borrero, 2017).

Para el caso de Colombia, el acuerdo de paz no consideró los bosques como un factor central durante el proceso de negociación (Murillo-Sandoval et al., 2020). La consecuencia de esto es que la deforestación, como lo indica Morales (2016), refleja la idea de que el ser humano tiene poder sobre la naturaleza, lo que ha impulsado que grupos al margen de la ley, mediante el empleo de colonos agricultores a menudo desplazados por la violencia, hayan realizado la explotación de nuevas tierras para el cultivo de coca y la expansión ganadera (Clerici et al., 2018). A lo anterior se suman las dificultades para el ejercicio de las entidades que tienen como función la administración de los recursos naturales, ya que, como lo indica (Botero-García et al., 2019), no siempre pueden ingresar a los territorios los funcionarios encargados de salvaguardar las áreas protegidas amazónicas.

Las consecuencias del fenómeno de deforestación radican en el incremento de la fragmentación, la disminución de las áreas que mantienen y conectan el flujo genético a gran escala y el intercambio de biodiversidad (Clerici et al., 2018), lo cual es particularmente negativo para el municipio de La Macarena, que hace parte del AMEM, un área que, sin embargo, ha sido objeto de la más variada legislación ambiental a fin de protegerla (Espinosa, 2006), por ejemplo con la Sentencia 4360 de 2018 (Corte Suprema de Justicia, Sala de Casación Penal, STC4360-2018, 2018), que exhorta a todos los entes del Estado a incrementar acciones para contrarrestar la deforestación en el bioma amazónico. Esto, no obstante, agudiza el conflicto socioambiental que, si bien estaba enmarcado en figuras de protección, ahora tiene el agravante de considerar a la Amazonía como sujeto de derechos.

Frente a expuesto por las encuestas, se identificó que la actividad que más se desarrolla en el municipio de La Macarena es la ganadería a mediana escala, seguida de la agricultura con cultivos de yuca, plátano, arroz y caña, resultados que coinciden con los presentados por CORMACARENA (2016): "en el municipio actualmente se destacan actividades de ganadería, ecoturismo, cultivo de plátano, yuca, maíz, arroz y cultivos ilícitos" (p. 30). Lo anterior confirma la premisa de que la transformación de áreas boscosas es la respuesta de la necesidad de los pobladores por satisfacer sus necesidades económicas inmediatas (Ruiz Serna, 2003). Según Carr (2003), existe de igual manera una relación entre la deforestación y la finalidad de establecimiento de cultivos como el maíz y el frijol o de territorios para el ganado de res.

Así, los factores o motores de deforestación después de los acuerdos de paz configuraron unas características particulares a raíz de la ausencia de figuras del Estado y la presencia de grupos al margen de la ley, lo que se confirmó en el proceso de levantamiento de información del presente estudio. A esto se suman las expectativas de titulación expuestas dentro de los mismos acuerdos bajo el nombre de "Formalización masiva de la pequeña y mediana propiedad rural", y la interrelación de algunas actividades económicas lícitas e ilícitas, como lo indican Murillo-Sandoval et al. (2020), quienes sostienen que el narcotráfico está fuertemente vinculado al acaparamiento de tierras para uso del ganado y producción de coca, actividades que requieren cada vez más extensiones. Lo anterior implica una inversión al momento de talar bosques y el ingreso a nuevas economías, lo que permite deducir que las comunidades campesinas no son los actores que promueven la deforestación. Por ello es que en su estudio Molano et al. (2013) resaltan la relación del fenómeno de colonización con prácticas como el cultivo de coca y la presencia de grupos al margen de la ley. 
Un factor que persiste como motor de deforestación es la presencia de vías (Mena et al., 2006; Murillo-Sandoval et al., 2020) que, para el caso de La Macarena, aparte de realizarse en áreas incompatibles para su desarrollo, es el resultado del control ejercido por grupos ilegales (BoteroGarcía et al., 2019).

En cuanto a la actividad de cultivos ilícitos, las encuestas muestran un porcentaje del $4.08 \%$ que la ubica en el cuarto renglón, después de la ganadería, la agricultura y la extracción de madera. De esta manera se evidencia que, al igual que la deforestación, el cultivo de coca es una actividad practicada en el municipio, dado que, conforme a lo expuesto por Puentes (2019), los cultivos ilícitos se concentran en los municipios del sur del departamento. Si bien las encuestas arrojaron un porcentaje bajo de esta actividad, el Meta y el Guaviare son regiones que históricamente han mantenido una alta concentración de cultivos de coca (SIMCl y UNODC, 2018) vinculados a actividades legales e ilegales, al acaparamiento de tierras y a la ganadería.

Entre las alternativas frente a la deforestación, Núñez (1992) reconoce el problema de los derechos de propiedad e indica que se debe profundizar en la incorporación del capital natural como activos económicos en el país. Por otra parte, Minaverry (2013) expone la necesidad de crear programas referidos al manejo de los recursos forestales, acompañados de políticas efectivas y educación ambiental, estimando que así se reducirían las tasas de deforestación y mejoraría la calidad de vida de las comunidades asociadas a los bosques. Por su lado, Ramos y García (2012) recomiendan que en colegios y escuelas se generen mayores conocimientos relacionados con la temática de la deforestación. Otras estrategias a nivel territorial adelantadas para contrarrestar la deforestación por parte de las entidades encargadas de administrar los recursos naturales en estas áreas, son: abarcar medidas de articulación interinstitucional entre la corporación ambiental y los PNN, promover estrategias comunitarias como el
Pago por Servicios Ambientales e incrementar la cobertura vegetal.

Los programas educativos se complementan con lo expuesto por Maya (1991), quien indica que es indispensable encontrar un camino para explicar la relación entre el ser humano y el sistema natural, debido a que la naturaleza ya se encuentra intervenida y el hombre no actúa como un individuo independiente y libre de contacto con lo que lo rodea. No obstante, se hace preciso conocer las dinámicas propias de la zona y evaluar los cambios en la diversidad, midiendo la configuración de agrosistemas y el grado de conectividad con los hábitats naturales contiguos antes de iniciar con cualquier iniciativa enfocada a la reducción de la deforestación (León, 2014). De ahí que González y Riascos (2007) señalen la necesidad de encontrar mecanismos para fortalecer el reconocimiento de las realidades del territorio y de los actores involucrados.

Por último, dada la complejidad de la zona estudiada en términos del componente social y político, sumado a que hace parte de una reserva biológica de la humanidad, se deduce que los modelos de protección de los PNN y de los DMI no son suficientes para garantizar la protección de ecosistemas tan importantes como los presentes en el municipio de La Macarena. Por tanto, como lo indican Sollund et al. (2019), las medidas a tomar para detener las tasas de deforestación deben concretarse antes de que continúe la pérdida de más ecosistemas claves. Así mismo, sería adecuado emplear la conservación inclusiva como un mecanismo a ejecutar cuando se presenten conflictos entre las comunidades por el manejo de las áreas (De Pourcq et al., 2017).

\section{CONCLUSIONES}

En los años posteriores a los acuerdos de paz, en el municipio de La Macarena se generó un aumento de deforestación. Al comparar los dos años previos y los dos años siguientes a la firma de los acuerdos, se identificó que es oportuna la aplicación de la fórmula de porcentaje de incremento de deforestación 
(\%ID) ya que, al estar dada en términos de cobertura perdida, evidenció que los cambios en dinámicas del territorio como resultado de la firma de los acuerdos de paz repercutieron en la aceleración de la pérdida de cobertura forestal.

Este proceso acelerado de pérdida de bosque natural incide directamente en la disminución de conectividad para el AMEM, así como en una baja de aportes ecosistémicos. Sin embargo, aunque hay una relación entre la firma de los acuerdos de paz y la deforestación, esta no es directa, dada la multiplicidad de factores como la presencia en el territorio de disidencias de las FARC-EP y el contexto histórico-social de necesidades insatisfechas, de violencia y de ausencia de figuras del Estado.

De las cuatro áreas del AMEM presentes en el municipio de La Macarena, la que ha sufrido mayor pérdida de bosque natural es el PNN Tinigua, donde se observa un incremento en deforestación del $1176.1 \%$ y que tiene como agravante el ser un área protegida perteneciente al bioma amazónico, sujeto de derechos desde 2018; el DMI Ariari Guayabero le sigue con un incremento de deforestación de $158.5 \%$.

Este incremento de deforestación en el municipio de La Macarena después de los acuerdos de paz se convierte en una externalidad negativa, más que de los acuerdos, de la falta de capacidad de respuesta para su implementación. El desmedido uso de los recursos naturales evidencia el poco arraigo por el territorio y resalta problemas coyunturales que se han presentado históricamente en el área y que no han sido atendidos de manera adecuada, lo que deriva en una disminución de aportes ecosistémicos del bosque e incrementa la fragmentación en esta reserva biológica de la humanidad. Por tanto, la deforestación no debe ser vista como un problema netamente ambiental sino de manera integral, incluyendo el contexto sociopolítico.

\section{AGRADECIMIENTOS}

A la Universidad de Manizales y la Corporación para el Desarrollo Sostenible del Área de Manejo
Especial La Macarena -CORMACARENA. También a Adriana Forero, Sara Lorena Pérez, Diego Barrios, Johan Ramírez, Héctor Fabio Almario (Q.E.P.D.), Andrés Acosta y Lorena Pinzón.

\section{CONFLICTOS DE INTERESES}

Los autores declaran no tener conflicto de intereses.

\section{CONTRIBUCIÓN POR EL AUTOR}

Los autores son los únicos responsables de la obra en todos los aspectos que condujeron a la elaboración de su publicación. J.F. ideó la investigación, llevó a cabo la misma en terreno y realizó el análisis de datos de deforestación. J.F. y M.P. contribuyeron a la discusión y comentaron los borradores.

\section{REFERENCIAS}

Andrade Correa, M. (2011). Estado del conocimiento de la biodiversidad en Colombia y sus amenazas. Consideraciones para fortalecer la interacción ciencia-política. Revista de la Academia Colombiana de Ciencias Exactas, Físicas y Naturales, 35(137), 491-508.

Andrade, G. I. y Castro, L. G. (2012). Degradación, pérdida y transformación de la biodiversidad continental en Colombia: invitación a una interpretación socioecológica. Ambiente y Desarrollo, 16(30), 53-54.

Armenteras, D., Rudas, G., Rodríguez, N., Sua, S. y Romero, M. (2006). Patterns and causes of deforestation in the Colombian Amazon. Ecological Indicators, 6(2), 353-368.

https://doi.org/10.1016/j.ecolind.2005.03.014

Armenteras, D., Schneider, L. y Dávalos, L. M. (2019). Fires in protected areas reveal unforeseen costs of Colombian peace. Nature Ecology and Evolution, $3(1), 20-23$.

https://doi.org/10.1038/s41559-018-0727-8 
Blanco-Libreros, J. F., Taborda-Marín, A., Amortegui-Torres, V., Arroyave-Rincón, A., Estrada, E. A. y Leal-Flórez, J. (2013). Deforestación y sedimetación en los manglares del Golfo de Urabá. Gestión y Ambiente, 16(2), 19-36.

Borrero, W. (2017). Análisis contextual de los posibles efectos en la deforestación de Cartagena del Chairá, Caquetá, luego del Acuerdo de Paz firmado con la Farc-EP a partir de experiencias internacionales (Tesis de Maestría), Universidad Distrital Francisco José De Caldas]. Repositorio Universidad Distrital Francisco José De Caldas.

https://repository.udistrital.edu.co/handle/11349 /7627

Botero-García, R., López, F., Ospino, H., Ponce de León-Chaux, E. y Riveros, C. (2019). Áreas protegidas amazónicas y sus funcionarios como víctimas del conflicto armado. Fundación para la Conservación y el Desarrollo Sostenible.

https://fcds.org.co/wp-content/uploads/2019/06/libro-areas-protegidas-amazonicas.pdf

Cabrera, E., Vargas, D. M., Galindo, G., García, M. C. y Ordoñez, M. F. (2011). Protocolo de procesamiento digital de imágenes para la cuantificación de la deforestación en Colombia, Nivel Nacional Escala gruesa y fina. IDEAM.

http://www.ideam.gov.co/documents/13257/13 817/Protocolo_para_la_cuantificación_Deforesta ción_Nivel_Nacional.pdf

Cárdenas-Torres, M. A. (2006). Estimación de la deforestación por cultivos ilícitos en la zona de reserva forestal del Río Magdalena. Colombia Forestal, 9(19), 136-154.

Carr, D. L. (2003). Migración rural-rural y deforestación en la Reserva de la Biosfera Maya, Guatemala. Método de entrevistas. Tiempos de América, 10, 19-27.

Clerici, N., Salazar, C., Pardo-Díaz, C., Jiggins, C. D., Richardson, J. E. y Linares, M. (2018). Peace in Colombia is a critical moment for Neotropical connectivity and conservation: save the northern Andes-Amazon biodiversity bridge. Conservation Letters, 12(1), 1-7.

CORMACARENA. Corporación para el Desarrollo Sostenible del Área de Manejo Especial La Macarena. (2016). Identificación de acciones tempranas para disminuir la deforestación y degradación de bosques al sur del Departamento del Meta.

Corte Suprema de Justicia. Sala de Casación Penal. (05 de abril de 2018). Sentencia STC4360-2018. (MP Luis Armando Tolosa Villabona).

https://www.cortesuprema.gov.co/corte/wp-content/uploads/2018/04/STC4360-2018-201800319-011.pdf

De Pourcq, K., Thomas, E., Van Damme, P. y Léon-Sicard, T. (2017). Análisis de los conflictos entre comunidades locales y autoridades de conservación en Colombia. Causas y recomendaciones. Gestión y Ambiente, 20(1), 122-139.

https://doi.org/10.15446/ga.v20n1.71174

Documento CONPES 4007 de 2020 [Consejo Nacional de Política Económica y Social - CONPES]. Estrategia para el Fortalecimiento de la Gobernanza en el Sistema de Administración del Territorio. 26 de octubre de 2020.

http://revistas.unal.edu.co/index.php/gestion/ article/view/1417

Documento CONPES 4021 de 2020 [Consejo Nacional de Política Económica y Social - CONPES]. Política Nacional para el Control de la Deforestación y la Gestión Sostenible de los Bosques. 21 de diciembre de 2020.

https://colaboracion.dnp.gov.co/CDT/Conpes/ Econ\%C3\%B3micos/4021.pdf

Espinosa, M. (2006). Violencia y vida campesina: reconstrucción etnográfica de la violencia de la vida diaria en zonas rurales de la Sierra de la Macarena. Revista Colombiana de Sociología, 27, 151-168.

Etter, A., Mcalpine, C., Phinn, S., Pullar, D. y Possingham, H. (2006). Characterizing a tropical deforestation wave: a dynamic spatial analysis of a deforestation hotspot in the Colombian Amazon. Global Change Biology, 12(8), 1409-1420.

https://doi.org/10.1111/j.1365-2486.2006.01168.x

FAO. Organización de las Naciones Unidas para la Agricultura y la Alimentación. (1996). Forest resources assessment 1990. Survey of tropical forest cover and study of change processes. http://www.fao.org/3/w0015e/W0015E00.htm 
Galindo, G., Espejo, O. J., Rubiano, J. C., Vergara, L. K., Cabrera, E. (2014). Protocolo de procesamiento digital de imágenes para la cuantificación de la deforestación en Colombia. V 2.0. IDEAM.

http://www.ideam.gov.co/documents/11769 /44688974/Protocolo+de+PDI+para+la+cuantificacion+de+la+deforestacion+en+colombia+v2_1_.pdf/00b95004-53dd-49f9-ab09-16d88 03ccd92? version $=1.0$

Gobierno Nacional y FARC-EP. (2016). Acuerdo Final para la Terminación del Conflicto y la Construcción de una Paz Estable y Duradera.

http://www.altocomisionadoparalapaz.gov.co/procesos-y-conversaciones/Documentoscompartidos/24-11-2016NuevoAcuerdoFinal.pdf

González, Á. y Riascos A. E. (2007). Panorama latinoamericano del pago por servicios ambientales. Gestión y Ambiente, 10(2), 129-144.

IDEAM. Instituto de Hidrología, Meteorología y Estudios Ambientales de Colombia. (2002). Tasa media anual de deforestación. En Primera generación de indicadores de la línea base de la información ambiental de Colombia (Tomo 2), pp. 764-769. IDEAM.

IDEAM [Instituto de Hidrología, Meteorología y Estudios Ambientales de Colombia] y MADS [Ministerio de Ambiente y Desarrollo Sostenible]. (2017). Estrategia integral de control a la deforestación. Actualización de cifras de monitoreo de bosques 2016.

http://www.ideam.gov.co/documents/24277/0/ Presentaci $\% 97 \mathrm{n}+$ Estrategia +Integral +de+Control+a+la+Deforestaci\%97n/173f79bf-3e68-4cbc9387-80123d09b5e2

IDEAM [Instituto de Hidrología, Meteorología y Estudios Ambientales de Colombia] y MADS [Ministerio de Ambiente y Desarrollo Sostenible]. (2018). Resultados Monitoreo de la Deforestación 2017. http://www.ideam.gov.co/documents/24 277/72115631/Actualizacion_cifras2017+FINAL. pdf/40bc4bb3-370c-4639-91ee-e4c6cea97a07

IDEAM [Instituto de Hidrología, Meteorología y Estudios Ambientales de Colombia] y MADS [Ministerio de Ambiente y Desarrollo Sostenible]. (2019). Resultados Monitoreo de la Deforestación 2018.

http://www.ideam.gov.co/documents/24277/9121 3793/Actualizacion_cifras2018FINALDEFORES TACION.pdf/80b719d7-1bf6-4858-8fd3-b5ce192a2fdc

Granados-Martínez, C., Lasso, C. A., Núñez-Avellaneda, M. y Morales-Betancourt, M. A. (2018). Macroinvertebrados acuáticos de los ríos Guayabero medio, bajo Losada y bajo Duda, sierra de La Macarena, Meta, Colombia. En C. A. Lasso, M. A. Morales-Betancourt e I. D. Escobar-Martínez (Eds.), Biodiversidad de la sierra de La Macarena, Meta, Colombia. Parte I. Ríos Guayabero medio, bajo Losada y bajo Duda (pp. 97-120). Investigación de Recursos Biológicos Alexander von Humboldt. https://doi.org/10.21068/a2018n02

León, T. (2014). Perspectiva ambiental de la agroecología: la ciencia de los agroecosistemas. Universidad Nacional de Colombia.

Ley 52 de 1948. Por la cual se declara reserva nacional la sierra denominada La Macarena, en la Intendencia del Meta, y se crea la Estación Biológica José Jerónimo Triana. D. O. No. 26891.

http://www.suin-juriscol.gov.co/viewDocument. asp?ruta=Leyes/1606080

Idrobo, J. M. (1984). Defensa de La Macarena. Colombia: Ciencia y Tecnología, 2(1), 2/20.

Maya, A. A. (1991). Ciencia, cultura y medio ambiente. Cuadernos de Desarrollo Rural, 26, 101-106.

Mena, C. F., Bilsborrow, R. E. y McClain, M. E. (2006). Socioeconomic drivers of deforestation in the Northern Ecuadorian Amazon. Environmental Management, 37(6), 802-815.

https://doi.org/10.1007/s00267-003-0230-z

Minaverry, C. (2013). Las comunidades locales y su relación con el aprovechamiento sostenible de los recursos del bosque en Argentina. Gestión y Ambiente, 16(2), 49-60.

Molano, A., Fajardo, D., Carrizosa J. y Rozo F. (2013). Yo le digo una de las cosas: la colonización de la reserva de La Macarena. Revista Colombiana de Sociología, 1(1), 119-125. 
Monjardín-Armenta, S. A., Pacheco-Angulo, C. E., Plata-Rocha, W. y Corrales-Barraza, G. (2017). La deforestación y sus factores causales en el estado de Sinaloa, México. Madera y Bosques, 23(1), 7-22. https://doi.org/10.21829/myb.2017.2311482

Morales, G. (2016). La apropiación de la naturaleza como recurso. Una mirada reflexiva. Gestión y Ambiente, 19(1), 141-154.

Moreno, L. A., Andrade, G. I. y Ruíz-Contreras, L. F. (Eds.). (2016). Biodiversidad 2016. Estado y tendencias de la biodiversidad continental de Colombia. Instituto de Investigación de Recursos Biológicos Alexander von Humboldt.

http://repository.humboldt.org.co/handle/20.500. 11761/32962

Moreno, L. A., Andrade, G. I. y Goméz, M. F. (Eds.). (2019). Biodiversidad 2018. Estado y tendencias de la biodiversidad continental de Colombia. Instituto de Investigación de Recursos Biológicos Alexander von Humboldt.

http://repository.humboldt.org.co/handle/20.500. 11761/35365

Murillo-Sandoval, P. J., Van Dexter, K., Van Den Hoek, J., Wrathall, D. y Kennedy, R. (2020). The end of gunpoint conservation: forest disturbance after the Colombian peace agreement. Environmental Research Letters, 15(3).

https://doi.org/10.1088/1748-9326/ab6ae3

Núñez, J. (1992). Desarrollo sustentable: un análisis en el sector forestal chileno. Estudios de Economía, 19, 257-276.

Pozzobon, E. N. y Osorio, R. A. (2002). Evaluación de las deforestaciones en la Reserva Forestal de Ticoporo, estado Barinas-Venezuela, en base al análisis multitemporal de imágenes de percepción remota. Revista geográfica venezolana, 43(2), 215-235.

Puentes, J. (25 de enero de 2019). La sustitución va lenta en uno de los municipios donde más se sembró coca. Semana Rural.

https://semanarural.com/web/articulo/sustitucion-coca-pnis-meta-parques-nacionales/795
Puyravaud, J. (2003). Standardizing the calculation of the annual rate of deforestation. Forest Ecology and Management, 177, 593-596.

Ramos, C. y García, M. R. (2012). Estudio de percepción de la problemática ambiental en Arauca. Revista Gestión y Ambiente, 15(1), 119-128.

Rodríguez S, L. (2007). Protocolo de Kyoto: debate sobre ambiente y desarrollo en las discusiones sobre cambio climático. Gestión y Ambiente, 10(2), 119-128.

Ruiz Serna, D. (2003). Campesinos entre la selva, invasores de reservas. Tabula Rasa, 1, 183-210. https://doi.org/10.25058/20112742.n01.08

Ruiz, J., Cárdenas, W. y Baquero, C. (2011). Deforestación y dinámica del bosque secundario en la Amazonia Colombiana 1986-2000. Revista de La Academia Colombiana de Ciencias Exactas, Físicas y Naturales, 35(137), 531-545.

SIMCI [Sistema Integrado de Monitoreo de Cultivos Ilícitos] y UNODC [Oficina de las Naciones Unidas contra la Droga y el Delito]. (2018). Monitoreo de territorios afectados por cultivos ilícitos 2017 Colombia.

https://www.unodc.org/documents/crop-monitoring/Colombia/Colombia_Monitoreo_territorios_ afectados_cultivos_ilicitos_2017_Resumen.pdf

Sollund, R., Maldonado, A. y Brieva Rico, C. (2019). El acuerdo entre Noruega y Colombia para proteger los bosques tropicales y reducir el calentamiento global: ¿éxito o fracaso? Revista Crítica Penal y Poder, 16, 75-98.

UNODC [Oficina de las Naciones Unidas contra la Droga y el Delito] y MinJusticia [Ministerio de Justicia y del Derecho]. (2015). Atlas de la caracterización regional de la problemática asociada a las drogas ilícitas en el departamento de Meta.

http://201.217.213.202/Portals/1/politica-regional/ Docs/2016/REATLAS43_meta.pdf

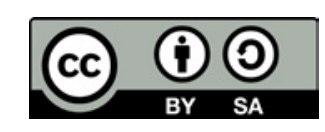

Colombia Forestal •ISSN 0120-0739 • e-ISSN 2256-201X • Bogotá-Colombia • Vol. 24 No. 2 • Julio-Diciembre de 2021 •pp. 9-23 